\title{
Palatalização na fala e na leitura de universitários sergipanos
}

\author{
Lucas Santos Silva ${ }^{1}$, Raquel Meister Ko. Freitag ${ }^{2}$ \\ ${ }^{1}$ Programa de Pós-Graduação em Letras - Centro de Educação e Ciências Humanas \\ Universidade Federal de Sergipe (UFS) - São Cristóvão - SE - Brazil \\ ${ }^{2}$ Departamento de Letras Vernáculas - Centro de Educação e Ciências Humanas \\ Universidade Federal de Sergipe (UFS) - São Cristóvão - SE - Brazil \\ lucas_riachao@hotmail.com, rkofreitag@academico.ufs.br
}

\begin{abstract}
A comparative analysis of the palatalization of $/ t /$ and $/ d /$ in two independent and different-sized samples, composed of speech $(n=8.850)$ and readaloud $(n=831)$ data from students at the Universidade Federal de Sergipe, is presented. Social variables displacement and time in the course, and linguistic variables anterior context, posterior context, tonicity, and sonority, were controlled in order to identifying whether the constraining effects of palatalization in speech also act on reading aloud data. The results point out that, even in distinct samples, the behavior of social and linguistic constrains shows stability in the change and propagation of palatalization process in the community.
\end{abstract}

Resumo. Apresentamos uma análise comparativa acerca da palatalização de $/ \mathrm{t} / \mathrm{e} / \mathrm{d} / \mathrm{em}$ duas amostras independentes, e com tamanho distinto, compostas por dados de fala $(n=8.850)$ e de leitura em voz alta $(n=831)$ de estudantes da Universidade Federal de Sergipe. Controlamos as variáveis sociais deslocamento e tempo no curso, e as variáveis linguísticas contexto anterior, contexto posterior, tonicidade e sonoridade, com o objetivo de identificar se os efeitos condicionantes da palatalização da fala atuam na leitura em voz alta. Os resultados sinalizam que, mesmo em amostras distintas, o comportamento dos fatores sociais e linguísticos apresentam estabilidade na mudança e propagação da palatalização na comunidade.

\section{Introdução}

A palatalização regressiva de /t/ e /d/, como em tia[t]ia] ou dia[dżia], é um traço fonético-fonológico que, no estado de Sergipe, passa por mudança incipiente com o aumento das variantes palatalizadas. O fenômeno foi descrito tanto em amostra de fala espontânea [Souza Neto 2008, Souza 2016, Freitag and Souza 2016, Corrêa 2019, Freitag et al. 2019], quanto em leitura em voz alta, com estudantes da educação básica [Pinheiro et al. 2017], assim como em estudos de percepção [Freitag and Santos 2016, Freitag 2020]. Os mesmos estudos apontam para o efeito dialetal condicionador da variação, com diferença na frequência entre moradores da capital sergipana, sendo o processo da palatalização mais frequente na capital [Souza 2016, Corrêa 2019].

Processos fonológicos da fala podem ser transpostos para a leitura em voz alta [Freitag and Sá 2019, Souza et al. 2020, Pinheiro et al. 2017] A identificação do fenômeno da palatalização na leitura em voz alta sinaliza que a variável emergente - a realização palatal - não é estigmatizada na comunidade. No escopo do projeto Como 
fala, lê e escreve o universitário?, a fim de identificar se condicionadores internos e externos da palatalização em contextos da fala atuam na leitura em voz alta, controlamos as variáveis sociais deslocamento e tempo de curso, e as variáveis linguísticas contexto anterior, contexto posterior, tonicidade e sonoridade em dois conjuntos de dados: uma amostra composta pela leitura em voz alta por 36 estudantes universitários sergipanos [Silva 2021], e o conjunto de dados de palatalização de uma amostra composta por entrevistas sociolinguísticas com 64 estudantes universitários sergipanos [Corrêa 2019].

A comparação dos condicionadores pode contribuir para identificar padrões na fala e na leitura em voz alta para a emergência de fenômenos variáveis no português brasileiro, ampliando o escopo de técnicas de coleta de dados.

\section{Método}

O conjunto dos dados de palatalização na fala espontânea é composto por 8.550 contextos de /t/ e /d/ diante de /i/ [Corrêa 2019]. Destas observações, 7.543 (88,2\%) referem-se à realização oclusiva. O conjunto dos dados de leitura em voz alta é composto por 831 contextos de /t/ e /d/ diante de /i/, dos quais 424 (51,02\%) referem-se à realização oclusiva [Silva 2021]. Ambos os conjuntos de dados provêm de uma mesma comunidade de fala, a de estudantes universitários da Universidade Federal de Sergipe, estratificados quanto ao tempo do curso, ao sexo e ao tipo de deslocamento que realizam: D1, D2, D3.

- Deslocamento 1 é composto por estudantes que residem na grande Aracaju;

- Deslocamento 2 é composto por estudantes residentes do interior do estado de Sergipe (nascidos e criados) e que fazem o movimento pendular diário para a UFS;

- Deslocamento 3 é composto por estudantes nascidos e criados no interior do estado de Sergipe, mas que moram na grande Aracaju por causa de UFS.

As amostras são independentes, e com tamanho distinto. Para poder realizar a comparação dos efeitos condicionantes, foi calculada a taxa de realização da variável dependente por falante, na fala espotânea e na leitura em voz alta, considerando o estudande como unidade de análise. Utilizamos o teste de Wilcoxon para amostras não pareadas. Primeiro, consideramos a frequência em cada variável, e depois, em cada variável, frequência e estilo, com ajuste de Bonferroni para ambos os casos. Os gráficos foram produzidos no R [Wickham et al. 2019, Wickham 2016, Kassambara 2020a, Kassambara 2020b].

\section{Resultados}

\subsection{Condicionamentos sociais}

Considerando os deslocamentos dos universitários sergipanos, não há diferença estatisticamente significativa entre a taxa média de palatalização na fala e na leitura em voz alta (Figura 1).

Os estudantes que têm maior contato com a região da grande a Aracaju (D1 e D2) tendem a fazer maior uso das variantes palatalizadas tanto na fala quanto na leitura em voz alta. A comparação em cada um dos níveis de deslocamos entre a taxa de palatalização na fala e na leitura em voz alta é estatisticamente significativa. Os universitários oriundos do 


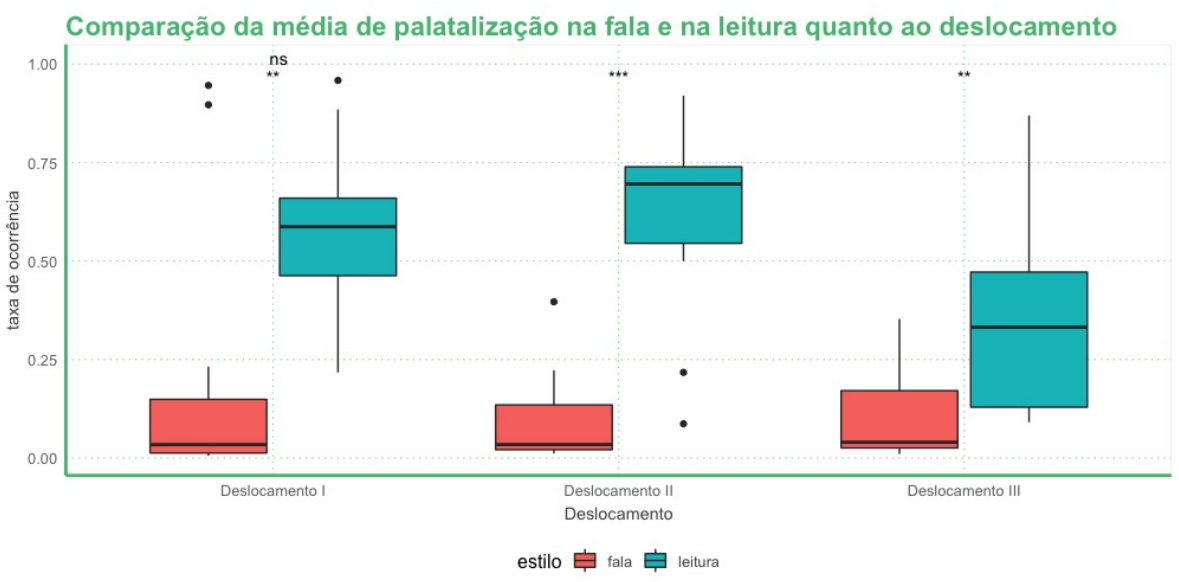

Figura 1. Comparação entre a taxa de realização de palatalização na fala e na leitura quanto ao deslocamento, com teste de Wilcoxon para o estilo, e comparação pareada entre os níveis

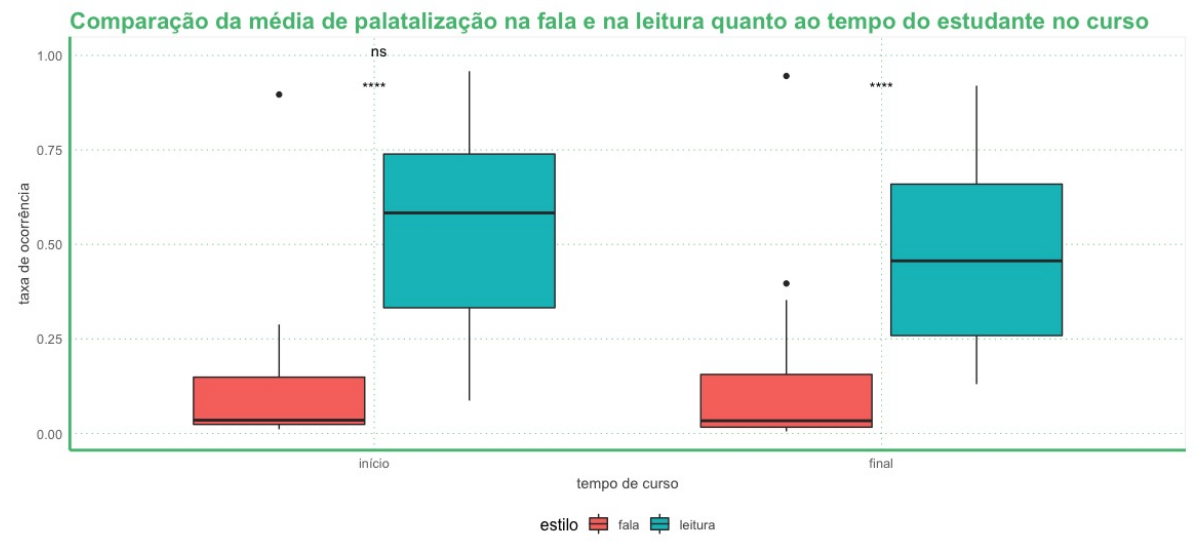

Figura 2. Comparação entre a taxa de realização de palatalização na fala e na leitura quanto ao tempo de curso, com teste de Wilcoxon para o estilo, e comparação pareada entre os níveis

interior do estado de Sergipe que foram morar na região da grande Aracaju apresentam a menor média na taxa de palatalização.

O tempo de curso pressupõe que o contato com a norma da comunidade pode afetar o padrão de uso da palatalização: universitários ao final do curso tendem a ter um comportamento linguístico diferente daqueles do início do curso. A comparação entre as taxas de palatalização na fala e na leitura em voz alta considerando o tempo de curso do estudante não se mostrou estatisticamente significativa (Figura 2). O tempo do curso não interfere na palatalização na leitura em voz alta. Mesmo não havendo diferença estatisticamente significativa, pelos valores das médias da taxa de ocorrência da palatalização, podemos inferir que os universitários do início do curso são os que fazem o maior uso das realizações palatalizadas. Novamente, há diferença estatisticamente significativa na comparação entre a fala e a leitura em cada um dos níveis da variável. 


\subsection{Condicionamentos linguísticos}

O contexto linguístico antecedente ao ambiente apresenta diferença estatisticamente significativa na comparação entre a fala e a leitura em voz alta (Figura 3). Nos contextos de fricativas alveolares, a exemplo de nos dizia [nuzdzi'ziə], se tivéssemos [s t $\int \mathrm{i}^{\prime} \mathrm{v} v \operatorname{sim} \mho \mathrm{s}$ ], as médias na taxa de palatalização na fala e na leitura em voz alta foram, respectivamente, 0,67 e 0,75, sem diferença estatisticamente significativa. Nos demais contextos, houve diferença estatisticamente significativa entre as médias de palatalização na leitura, todas superiores às encontradas na fala: fricativas alveolopalatais (tivéssemos de [ti'vesim\}z'dzI]) e glotais (morte ['moht $\left.\int \mathrm{I}\right]$ ); vogais anteriores (que tinham [ki 't $\left.\mathrm{fij} \tilde{e} v\right]$ ) e vogais centrais (atiraríamos [a.t]ira'riãmus]).

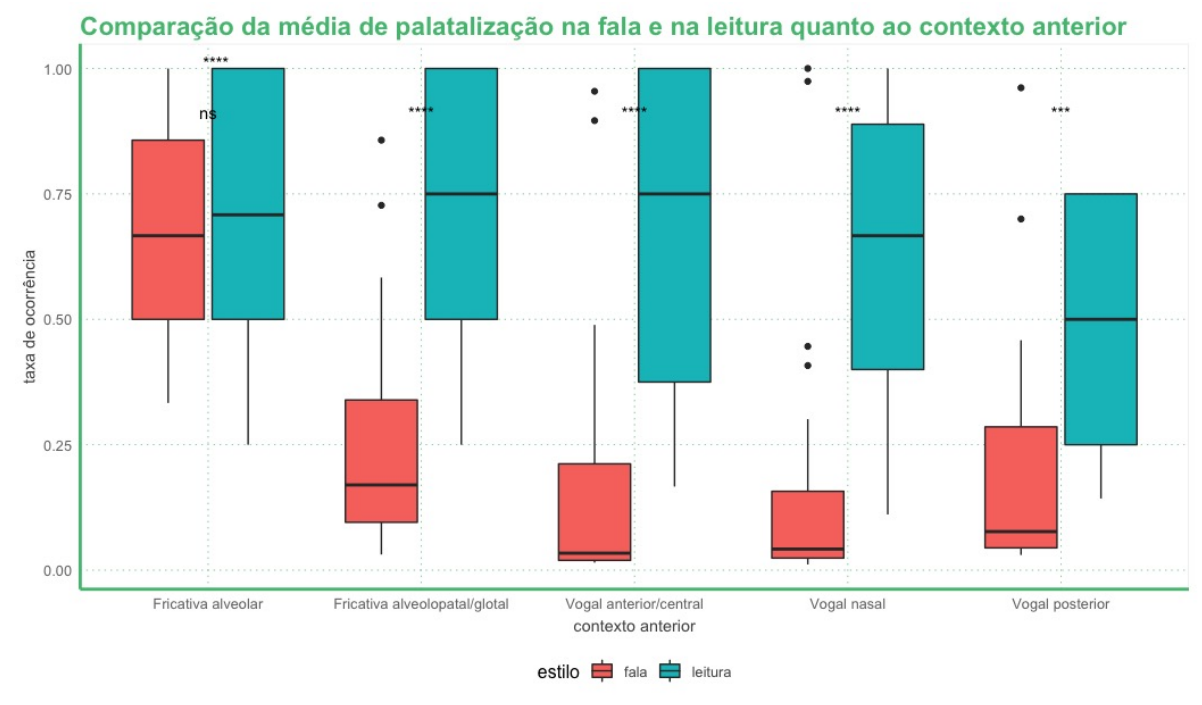

Figura 3. Comparação entre a taxa de realização de palatalização na fala e na leitura quanto ao contexto linguístico anterior, com teste de Wilcoxon para o estilo, e comparação pareada entre os níveis

Mesmo padrão de comportamento identificado no contexto linguístico sucedente ao ambiente, que também apresenta diferença estatisicamente significativa na comparação entre fala e leitura em voz alta (Figura 4). A diferença entre as médias de palatalização entre fala e leitura é mais acentuada nos ambientes seguidos por pausa (balde ['bawdzi]) e por vogal (apaixonamentente e... [aparfõnada'mẽt $\int \mathrm{I}$ e]), do que nos ambientes seguidos por consoante líquida ou fricativa (atiraríamos [atfira'riãmos] e utilizando [utfili'zãdv]) e consoante oclusiva/nasal (tipo ['tıpv] e continham [kõtfipuão]). O resultado segue o continuum para a mudança e propagação da palatalização, na fala e na leitura em voz alta, primeiro sendo as vogais e pausa em contexto seguinte as favorecedoras para a emergência do fenômeno em Sergipe [Corrêa 2019].

Quanto à tonicidade e à sonoridade, não há diferença estatisticamente significativa na taxa de palatalização na fala e na leitura. Na comparação entre os níveis de tonicidade (Figura 5), as médias na taxa de palatalização na leitura são superiores às da fala no postônico final (morte, apaixonadamente, balde, pode), pretônico (continuam, antigamente, tivéssemos, dizia) e tônico (iludindo, ditas, tinham). As médias na taxa de palatalização na fala e na leitura em voz alta são maiores no contexto tônico, corroborando a hipótese de que a emergência da palatalização se dá em contexto de sílaba tônica 


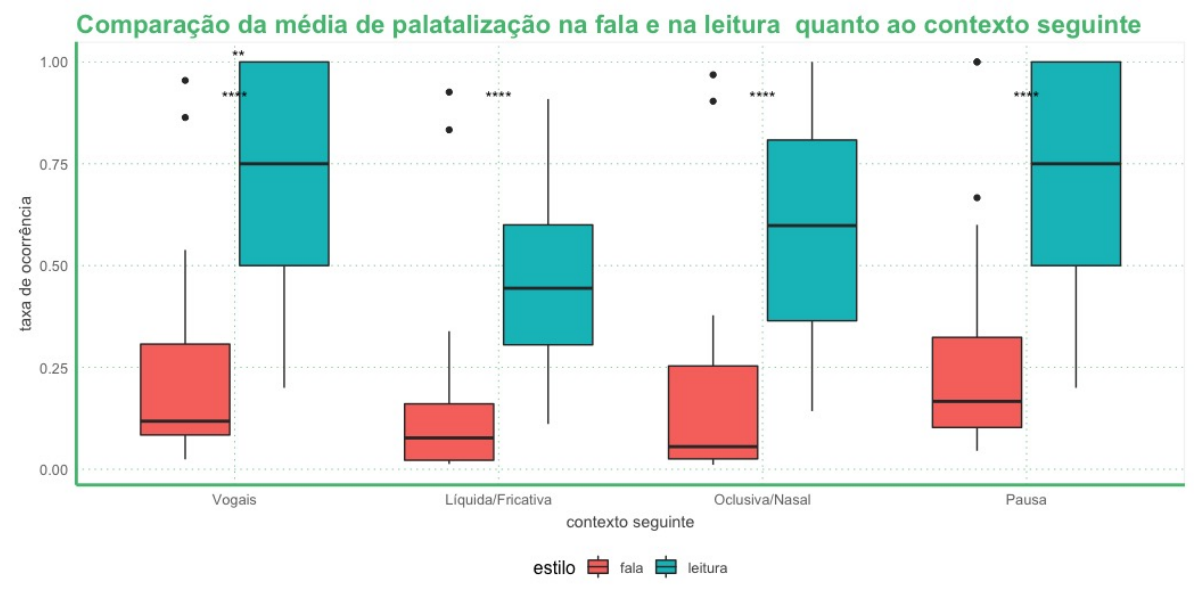

Figura 4. Comparação entre a taxa de realização de palatalização na fala e na leitura quanto ao contexto linguístico posterior, com teste de Wilcoxon para o estilo, e comparação pareada entre os níveis

[Câmara 1970].

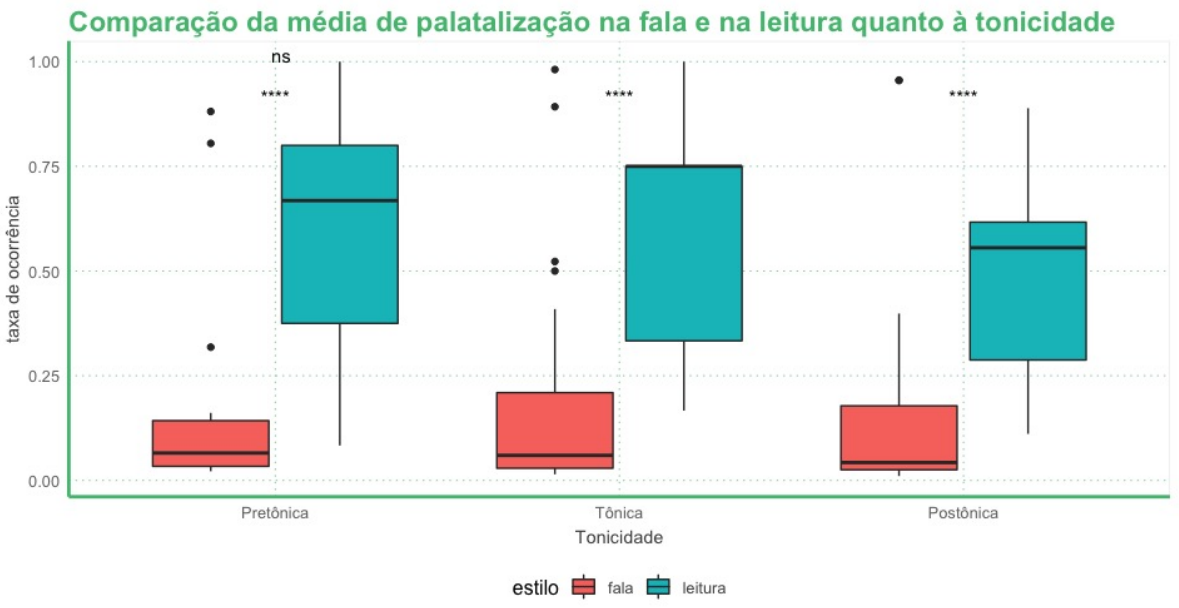

Figura 5. Comparação entre a taxa de realização de palatalização na fala e na leitura quanto à tonicidade da sílaba, com teste de Wilcoxon para o estilo, e comparação pareada entre os níveis

A diferença entre as amostras de fala e de leitura não é estatisticamente significativa quanto à sonoridade da consoante (Figura 6). As médias na taxa de palatalização apresentam semelhança em ambas as amostras: na fala, média $=0,12$ para sonoras e média $=0,16$ para surdas; para a leitura, média $=0,48$ das sonoras e média $=0,59$ das surdas. Em ambas as amostras, a consoante surda /t/ apresenta maior média de palatalização.

\section{Discussão}

Os resultados apresentados apontam que, embora haja diferença nos valores de frequência, com médias maiores na leitura do que na fala, o comportamento dos fatores sociais e linguísticos controlados apresentam estabilidade. A realização palatal é uma variante que tem aumentado sistematicamente (não em progressão exponencial), sendo 


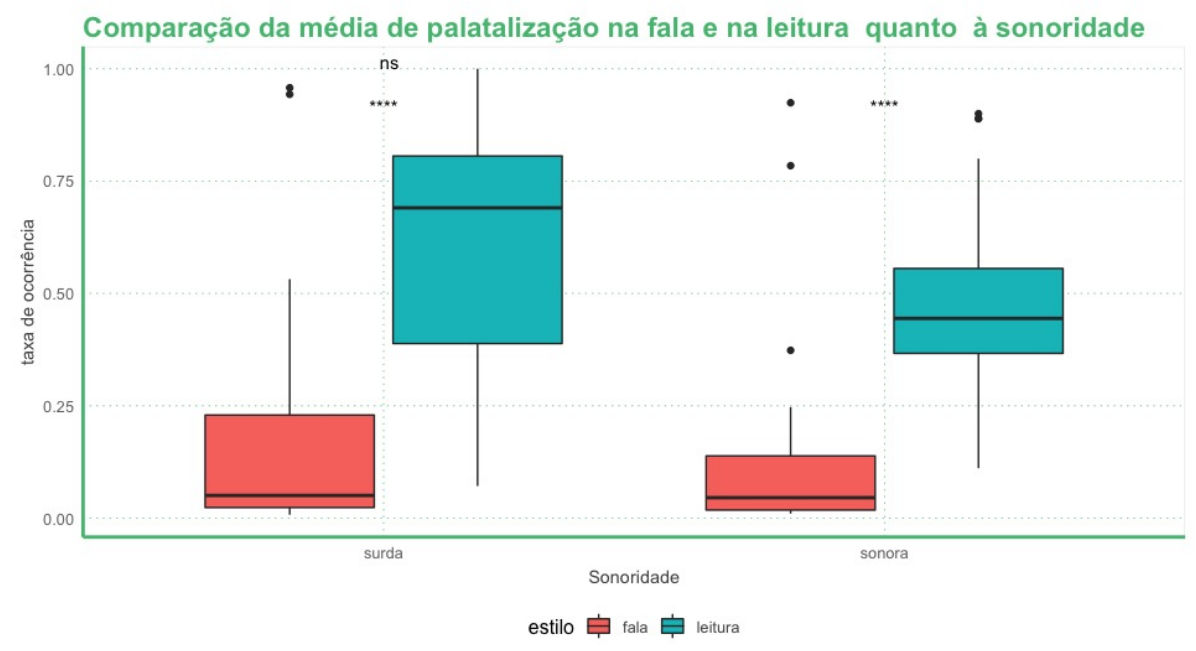

Figura 6. Comparação entre a taxa de realização de palatalização na fala e na leitura quanto à sonoridade da consoante, com teste de Wilcoxon para o estilo, e comparação pareada entre os níveis

socialmente prestigiada, conscientemente bem avaliada pela comunidade e associada aos falantes com alta escolaridade. Por isso, as maiores médias de frequência da palatalização na leitura em voz alta de universitários podem apresentar associação com o prestígio da variante e com o fato da tarefa de leitura ser altamente monitorada e consciente pelos estudantes.

Nas variáveis linguísticas, mesmo tendo diferenças estatisticamente significativas nos contextos seguinte e antecedente, os resultados são inconclusivos e não nos permitem fazer generalizações, uma vez que metodologias diferentes quanto aos dados de fala e de leitura foram adotadas. Para realizar a comparação, foi necessário realizar amalgamações entre níveis. Além disso, há menos itens lexicais nos dados de leitura ou itens sobrepostos: por exemplo, na leitura, a fricativa alveolar ocorre em seis contextos, dos quais cinco eram de consoante sonora, o que pode ter levado a uma diferença e não permite estabelecer uma generalização acerca da comparação entre os contextos. Tanto na fala quanto na leitura, o contexto das fricativas alveolares [s, z] é o que mais favorece a ocorrência de palatalização, resultado que segue a direção de outros estudos já desenvolvidos em Sergipe [Souza Neto 2008, Souza 2016, Corrêa 2019].

Ao receber alunos de diversas regiões geográficas de Sergipe, a Universidade $\mathrm{Fe}$ deral de Sergipe configura-se como um ambiente de troca de experiência. A inserção de universitários de outras comunidades do interior de Sergipe à grande Aracaju e, sobretudo, à Universidade Federal de Sergipe, contribui para o aumento da taxa de palatalização, uma vez que os estudantes assumem novas experiências linguísticas enquanto falantes.

No contexto altamente monitorado, como é o da leitura em voz alta, a média na taxa de realização de palatalização é maior em estudantes residentes no interior do estado de Sergipe, o que vai de encontro aos estudos já desenvolvidos acerca da palatalização em Sergipe com dados de fala e que apontam que o fenômeno da palatalização é mais frequente na grande Aracaju. Este resultado pode ser decorrente de hipercorreção, o que pode ser melhor explorado em outras abordagens. 


\section{Referências}

Câmara, J. M. (1970). Estrutura da língua portuguesa. Petrópolis, RJ: Editora Vozes.

Corrêa, T. R. d. A. (2019). A variação na realização de /t/ e /d/ na comunidade de práticas da UFS: mobilidade e integração. PhD thesis, Pós-Graduação em Letras, Universidade Federal de Sergipe.

Freitag, R. M. K. (2020). Effects of the linguistics processing: Palatals in brazilian portuguese and the sociolinguistic monitor. University of Pennsylvania Working Papers in Linguistics, 25(2):4.

Freitag, R. M. K. and Sá, J. J. D. S. (2019). Leitura em voz alta: variação linguística e o sucesso na aprendizagem inicial da leitura. Ilha do Desterro A Journal of English Language, Literatures in English and Cultural Studies, 72(3):41-62.

Freitag, R. M. K. and Santos, A. d. O. (2016). Percepção e atitudes linguísticas em relação às africadas pós-alveolares em sergipe. A Fala Nordestina: entre a sociolinguística e a dialetologia. São Paulo: Blucher, pages 109-122.

Freitag, R. M. K. and Souza, G. G. A. (2016). O caráter gradiente vs. discreto na palatalização de oclusivas em sergipe. Tabuleiro de Letras, 10(2):78-89.

Freitag, R. M. K., Souza Neto, A. F. d., and Corrêa, T. R. A. (2019). Panorama da palatalização em sergipe. Língua e sociedade: diferentes perspectivas, fim comum, page $63-80$.

Kassambara, A. (2020a). ggpubr: 'ggplot2' Based Publication Ready Plots. R package version 0.4.0.

Kassambara, A. (2020b). rstatix: Pipe-Friendly Framework for Basic Statistical Tests. R package version 0.6.0.

Pinheiro, B. F. M., Silva, L. S., Araújo, L. C., Quirino, R. R., Souza, V. R. A., and Freitag, R. M. K. (2017). Processos fonológicos que passam da fala para a leitura. Leitura, escrita e literatura: interseções e convergências. São Cristóvão, EdUFS, pages 10-25.

Silva, L. S. (2021). Análise acústica ou de oitiva: contribuições para o estudo da palatalização em Sergipe. PhD thesis, Pós-Graduação em Letras, Universidade Federal de Sergipe.

Souza, G. G. A. (2016). Palatalização de oclusivas alveolares em Sergipe. PhD thesis, Pós-Graduação em Letras: Universidade Federal de Sergipe.

Souza, V. R. A., Silva, V. L. S., and de Araujo Júnior, M. M. (2020). Da fala à leitura. Porto das Letras, 6(1):167-199.

Souza Neto, A. F. d. (2008). Realizações dos fonemas /t/ e /d/ em Aracaju, Sergipe. São Cristóvão: EdUFS.

Wickham, H. (2016). ggplot2: Elegant Graphics for Data Analysis. Springer-Verlag New York.

Wickham, H., Averick, M., Bryan, J., Chang, W., McGowan, L. D., François, R., Grolemund, G., Hayes, A., Henry, L., Hester, J., Kuhn, M., Pedersen, T. L., Miller, E., Bache, S. M., Müller, K., Ooms, J., Robinson, D., Seidel, D. P., Spinu, V., Takahashi, 
K., Vaughan, D., Wilke, C., Woo, K., and Yutani, H. (2019). Welcome to the tidyverse. Journal of Open Source Software, 4(43):1686. 